\title{
A marrow-minded look at immune checkpoint blockade resistance in metastatic castration resistant prostate cancer
}

\author{
Nicolette M. Fonseca^, Morgan E. Roberts, Alexander W. Wyatt^^ \\ Vancouver Prostate Centre, Department of Urologic Sciences, University of British Columbia, Vancouver, BC, Canada \\ Correspondence to: Dr. Alexander W. Wyatt. Vancouver Prostate Centre, Department of Urologic Sciences, University of British Columbia, 2660 Oak \\ Street, Vancouver, BC, V6H 3Z6, Canada. Email: awwyatt@mail.ubc.ca.
}

Submitted Aug 27, 2020. Accepted for publication Nov 30, 2020.

doi: $10.21037 /$ tau-20-1205

View this article at: http://dx.doi.org/10.21037/tau-20-1205

Prostate cancer is the second most common malignancy diagnosed in men and the sixth highest contributor to cancer-related mortality worldwide (1). Advanced prostate cancer initially responds to androgen deprivation therapy before progression to an incurable state termed metastatic castration resistant prostate cancer (mCRPC). mCRPC is typically treated with taxanes or androgen receptor (AR) pathway inhibitors, but the survival benefit is variable and often brief (2). In recent years, focus has turned to the development of therapies outside of the AR signaling pathway. PARP inhibitors and pembrolizumab are effective in the subset of mCRPC with homologous recombination repair and mismatch repair defects, respectively $(3,4)$. Further agents including PSMA-lutetium-177, DNA peptide-based vaccines, and oncolytic viruses are in various stages of clinical development (3). While single agent immune checkpoint blockade (ICB) has proved relatively ineffective in unselected mCRPC, in this commentary, we highlight its potential for eliciting potent anti-tumor responses when combined with agents that target the uniquely immunosuppressive tumor microenvironment (TME) of mCRPC.

In recent years, ICB has shown remarkable success in the treatment of some metastatic cancers including melanoma, lung, and urothelial carcinoma. Approved ICBs target either CTLA-4 or the PD-1/PD-L1 axis to promote tumor cell elimination by enhancing $\mathrm{T}$ cell activation and invigorating exhausted T cells in the TME. In metastatic melanoma, long-term disease control is possible via the generation of T cell memory in response to CTLA-4 blockade $(5,6)$. In prostate cancer, targeting the immune system initially gained traction with the FDA approval of Sipuleucel-T, an autologous cellular vaccine, for patients with mCRPC (7). However, subsequent phase III clinical trials employing ICB with ipilimumab (anti-CTLA-4), atezolizumab (antiPD-L1), autologous cellular (PROSTVAC) or viral vaccine (GVAX), and phase II trials with pembrolizumab (antiPD1) have shown no significant survival benefit $(8,9)$. Early results from CheckMate 650, an on-going phase II clinical trial employing a combination of nivolumab (anti-PD1) and ipilimumab (anti-CTLA-4), showed promising objective response rates of $26 \%$ in chemotherapy-naive patients, and $10 \%$ in chemotherapy-exposed patients. However, about half of patients experienced grade 3-5 adverse events, and a third discontinued treatment (10). Collectively, ICB clinical trial results and correlative analyses have indicated that most mCRPC is immunologically 'cold' and resistant to current immunotherapeutic approaches.

The poor response to ICB in prostate cancer is attributable to a combination of extrinsic and intrinsic resistance mechanisms including low mutational and neoantigen burden, defective MHC-I restricted antigen processing and presentation, paucity of inflammatory gene signatures, and the presence of immunosuppressive cell types $(11,12)$. In particular, myeloid derived suppressor cells (MDSCs) appear to have an important role in mCRPC progression as they secrete cytokines that suppress $\mathrm{T}$ cells, promote $\mathrm{M} 2$ macrophage polarization, and can also directly

^ ORCID: Nicolette M. Fonseca: 0000-0001-8529-4703; Alexander W. Wyatt: 0000-0003-2399-0329. 
promote AR-mediated tumor growth through IL-23/IL23R signaling (13). Treatment exposure likely further alters the immune microenvironment and contributes to ICB resistance. For example, increased M2 macrophages and Tregs have been observed in tumors or tumor draining lymph nodes of mice exposed to androgen deprivation therapy (14-16). Of course, prostate tumors are notoriously heterogeneous, and in a recent phase II trial of ipilimumab in $\mathrm{mCRPC}$, elevated $\mathrm{CD}^{+} \mathrm{T}$ cell infiltration and expression of an IFN $\gamma$ gene signature in pretreatment tumors were associated with disease control and survival benefit (17). Importantly, this suggests that there may be an opportunity to improve the efficacy of ICB in prostate cancer through a combination approach that targets the immunosuppressive TME and invigorates infiltrating $\mathrm{T}$ cells. One such approach, with demonstrated efficacy over single agent use in a preclinical mouse model, was the pairing of ICB with phosphokinase inhibitors which cooperatively decreased mCRPC tumor growth, by selectively targeting tumor infiltrating T cell-inhibitory MDSCs, and synergistically enhancing ICB induced $\mathrm{CD} 8^{+} \mathrm{T}$ cell expansion (18).

Bone is the favored site of metastatic lesions in mCRPC and appears to be uniquely suited to promoting prostate cancer growth. Indeed, the establishment of bone metastases fits with Paget's 'seed and soil' hypothesis as prostate cancer cells migrate by hijacking bone tropic chemokine cues meant for hematopoietic cells, invade the bone endosteum, and eventually colonize the bone through paracrine signaling processes involving tumor cells and bone-resident osteoblasts, osteoclasts, and other stromal cells (19). Bone metastases frequently form in the axial skeleton including the spine, pelvis and ribs. These skeletal locations also house the bone marrow which functions as an essential lymphoid organ containing hematopoietic stem and progenitor cells, as well as mature adaptive and innate immune cell lineages (20). Therefore, for successful metastatic colonization, prostate tumor cells must first establish a foothold in the marrow and alter the bone microenvironment to a pro-tumorigenic state. The presence of a variety of immune cells suggests that tumorimmune crosstalk in the bone microenvironment is an important factor in metastatic disease progression. In this regard, TGF- $\beta 1$ released by dysregulated osteoclastic bone resorption, stands out as a critical immunomodulator that can exert suppressive effects on antitumor immunity by inhibiting Th1 cells and cytotoxic CD8 T cells (21). Other examples of tumor-promoting interactions between bone metastases and immune cells include the recruitment of immunosuppressive Tregs, MDSCs and macrophages via tumor mediated chemokine signaling, and expression of osteoclast activating molecules by plasmacytoid dendritic cells (22). Taken together, it is plausible that tumorimmune cell crosstalk within the bone microenvironment contributes to immunotherapy resistance however this remains a relatively underexplored area.

The value of dissecting the bone immune microenvironment in mCRPC was thrown into the limelight by a recent groundbreaking study from Jiao and colleagues that identified and exploited an ICB resistance mechanism within the bone marrow of men with mCRPC (23). The work was predicated upon the observation that patients with bone metastases have worse outcomes in ICB trials than those without bone metastases (10,24). Examination of immune signatures in the bone and soft tissue metastases of ICB-treated patients revealed site-specific differences in the tumor-associated $\mathrm{T}$ cell compartment. In bone metastases, ipilimumab treatment led to an expansion of Th17 cells while visceral metastases exhibited a skew towards Th1 responses. This distinction in immune response is important because Th1 cells are thought to be better at generating and maintaining cytotoxic $\mathrm{CD} 8^{+} \mathrm{T}$ responses necessary for tumor clearance compared with Th17 cells. To validate these site-specific differences in Thelper response, the authors generated osseous and subcutaneous mCRPC animal models using a syngeneic Myc-CaP cell line and degarelix-induced castration. Consistent with the human data, when treated with a combination of anti-CTLA-4 and anti-PD1, animals with only subcutaneous tumors displayed increased survival and tumor control compared to mice with osseous tumors. Furthermore, single cell analysis of the immune infiltrates in osseous and subcutaneous tumors suggested that although ICB resulted in expanded $\mathrm{CD}^{+} \mathrm{T}$ cells, $\mathrm{CD}^{+} \mathrm{T}$ cells, and dendritic cells in both metastatic niches, the quality of the $\mathrm{CD}^{+} \mathrm{T}$ cell response differed considerably. Again mirroring human metastatic sites, Th17 and Treg cells dominated in the bone while Th1 cells expanded in subcutaneous tumors (23). Interestingly, this pattern of Th17/Treg $v s$. Th1 polarization also appeared to be present prior to ICB treatment, suggesting that ICB expands $\mathrm{T}$ cells in a lineage-constrained manner.

To investigate further, the authors interrogated the cytokine, chemokine, and growth factor profile of bone marrow in their model system. Bone metastases showed an increase in TGF- $\beta 1$ expression relative to normal contralateral bone from the same animal or relative to bone from healthy control animals. IL-6 was also altered in bone 
marrow but the elevated expression pattern was similar in both healthy and diseased bone compared to serum indicating that increased IL- 6 is merely a feature of the osteogenic bone environment. Nevertheless, TGF- $\beta 1$ and IL-6 are well-established synergistic promoters of Th17 polarization (25), and TGF- $\beta 1$ can also limit Th1 responses; thus providing a potential explanation for the inappropriate Th17 response observed in the ICB-exposed bone metastases of both animal models and humans. Elevated TGF- $\beta 1$ expression was tied to RANKL dependent bone resorption by osteoclasts, as RANKL blockade reduced osteoclastic activity and decreased TGF- $\beta 1$ expression. The increased TGF- $\beta 1$ expression observed in murine tumor bearing femurs was also confirmed in human mCRPC bone lesions (23).

The identification of elevated RANKL, IL- 6 and TGF- $\beta 1$ nominated several options for evaluation in combination with ICB to improve treatment outcomes. However, Jiao and colleagues found that anti-TGF- $\beta$ blockade synergized best with anti-CTLA-4 and anti-PD1 to control tumor growth and improve survival. Importantly, anti-TGF- $\beta$ could be combined with anti-CTLA-4 alone to provide similar therapeutic benefit, thereby highlighting a clinically-relevant pairing which may reduce adverse effects compared to dual ICB. Examination of immune cell composition in these single ICB and anti-TGF- $\beta$ treated osseous mCRPC tumors showed an expansion of Th1 cells and depletion of Tregs. Surprisingly, the Th17 compartment was still present suggesting that pre-existing Th17 cells were able to expand in a TGF- $\beta$ independent manner in response to treatment. IL- 6 and IL-23 are known to promote and maintain the Th17 lineage (26) and it is possible that osteoblasts or MDSCs secreting these cytokines in CRPC bone mets may continue to support Th17 cell development. Since cytotoxic T cells are the main tumor eliminating immune cell type, the authors investigated the effects of anti-TGF- $\beta$ and anti-CTLA- 4 alone or in combination on intratumoral CD8 T cells. T cell receptor diversity analysis showed that the proportion of expanded clones was highest in the context of combined anti-TGF- $\beta$ and anti-CTLA- 4 blockade (although only a few $\mathrm{T}$ cell clones showed evidence of expansion). These CD8 $\mathrm{T}$ cell clones possessed an effector/memory signature and expressed genes associated with $\mathrm{T}$ cell cytotoxicity and tissue retention (23). In summary, this study provided detailed insight into a TGF- $\beta$ linked bone-specific mechanism of mCRPC resistance to ICB, and demonstrated that targeting this immunosuppressive cytokine could move the needle from 'cold' to 'hot' in recalcitrant bone metastases.

TGF- $\beta$ signaling also appears to be a core pathway contributing ICB resistance in 'immune excluded' metastatic urothelial carcinomas and colorectal cancer $(27,28)$. Encouragingly, in preclinical studies, the TGF- $\beta$ signaling pathway can be targeted by anti-TGF- $\beta$ mediated depletion or by TGF- $\beta$ inhibitors such as galunisertib $(27,28)$. Together, these findings set the stage for clinical trials testing ICB in combination with TGF- $\beta$ inhibition in $\mathrm{mCRPC}$ and other cancers with immunosuppressive microenvironments. Of course, given the heterogeneity of prostate cancer, clinical implementation of this combination will likely require development of predictive biomarkers. Importantly, while Jiao et al. utilized a MYC-expressing mCRPC model system (23), human mCRPC comprises several distinct molecular subtypes, each with differential biological and clinical impact (29). As such, there is a need to understand whether underlying tumor genomic and transcriptomic subtypes are associated with the degree of immunosuppression in bone metastases. This may be particularly relevant in the context of AR expression heterogeneity, since about $20 \%$ of bone metastases appear to exhibit low AR activity, and higher concomitant immune cell infiltration and MHC-I expression (30).

\section{Acknowledgments}

Funding: None.

\section{Footnote}

Provenance and Peer Review: This article was commissioned by the Guest Editor (Tilman Todenhöfer) for the series "Management of Advanced Genitourinary Malignancies" published in Translational Andrology and Urology. The article has undergone external peer review.

Conflicts of Interest: All authors have completed the ICMJE uniform disclosure form (available at http://dx.doi. org/10.21037/tau-20-1205). The series "Management of Advanced Genitourinary Malignancies" was commissioned by the editorial office without any funding or sponsorship. Dr. AWW reports personal fees from AstraZeneca, personal fees from Astellas, grants and personal fees from Janssen, personal fees from Merck, outside the submitted work. The authors have no other conflicts of interest to declare.

Ethical Statement: The authors are accountable for all 
aspects of the work in ensuring that questions related to the accuracy or integrity of any part of the work are appropriately investigated and resolved.

Open Access Statement: This is an Open Access article distributed in accordance with the Creative Commons Attribution-NonCommercial-NoDerivs 4.0 International License (CC BY-NC-ND 4.0), which permits the noncommercial replication and distribution of the article with the strict proviso that no changes or edits are made and the original work is properly cited (including links to both the formal publication through the relevant DOI and the license). See: https://creativecommons.org/licenses/by-nc-nd/4.0/.

\section{References}

1. Bray F, Ferlay J, Soerjomataram I, et al. Global cancer statistics 2018: GLOBOCAN estimates of incidence and mortality worldwide for 36 cancers in 185 countries. CA Cancer J Clin 2018;68:394-424.

2. Sartor O, de Bono JS. Metastatic prostate cancer. N Engl J Med 2018;378:1653-4.

3. Marshall CH, Antonarakis ES. Emerging treatments for metastatic castration-resistant prostate cancer: immunotherapy, PARP inhibitors, and PSMA-targeted approaches. Cancer Treat Res Commun 2020;23:100164.

4. de Bono J, Mateo J, Fizazi K, et al. Olaparib for metastatic castration-resistant prostate cancer. $\mathrm{N}$ Engl J Med 2020;382:2091-102.

5. Schadendorf D, Hodi FS, Robert C, et al. Pooled analysis of long-term survival data from phase II and phase III trials of ipilimumab in unresectable or metastatic melanoma. J Clin Oncol 2015;33:1889-94.

6. Waldman AD, Fritz JM, Lenardo MJ. A guide to cancer immunotherapy: from $\mathrm{T}$ cell basic science to clinical practice. Nat Rev Immunol 2020;20:651-68.

7. Kantoff PW, Higano CS, Shore ND, et al. Sipuleucel-T immunotherapy for castration-resistant prostate cancer. $\mathrm{N}$ Engl J Med 2010;363:411-22.

8. Boettcher AN, Usman A, Morgans A, et al. Past, current, and future of immunotherapies for prostate cancer. Front Oncol 2019;9:884.

9. Sweeney CJ, Gillessen S, Rathkopf D, et al. Abstract CT014: IMbassador250: A phase III trial comparing atezolizumab with enzalutamide $v s$ enzalutamide alone in patients with metastatic castration-resistant prostate cancer (mCRPC). Cancer Res 2020;80:CT014.

10. Sharma P, Pachynski RK, Narayan V, et al. Initial results from a phase II study of nivolumab (NIVO) plus ipilimumab (IPI) for the treatment of metastatic castrationresistant prostate cancer (mCRPC; CheckMate 650). J Clin Oncol 2019;37:142.

11. Vitkin N, Nersesian S, Siemens DR, et al. The tumor immune contexture of prostate cancer. Front Immunol 2019;10:603.

12. Danaher P, Warren S, Lu R, et al. Pan-cancer adaptive immune resistance as defined by the Tumor Inflammation Signature (TIS): results from The Cancer Genome Atlas (TCGA). J Immunother Cancer 2018;6:63.

13. Calcinotto A, Spataro C, Zagato E, et al. IL-23 secreted by myeloid cells drives castration-resistant prostate cancer. Nature 2018;559:363-9.

14. Tang S, Moore ML, Grayson JM, et al. Increased CD8+ T-cell function following castration and immunization is countered by parallel expansion of regulatory $\mathrm{T}$ cells. Cancer Res 2012;72:1975-85.

15. Akins EJ, Moore ML, Tang S, et al. In situ vaccination combined with androgen ablation and regulatory T-cell depletion reduces castration-resistant tumor burden in prostate-specific Pten knockout mice. Cancer Res 2010;70:3473-82.

16. Escamilla J, Schokrpur S, Liu C, et al. CSF1 receptor targeting in prostate cancer reverses macrophage-mediated resistance to androgen blockade therapy. Cancer Res 2015;75:950-62.

17. Subudhi SK, Vence L, Zhao H, et al. Neoantigen responses, immune correlates, and favorable outcomes after ipilimumab treatment of patients with prostate cancer. Sci Transl Med Sci Transl Med 2020;12:eaaz3577.

18. Lu X, Horner JW, Paul E, et al. Effective combinatorial immunotherapy for castration-resistant prostate cancer. Nature 2017;543:728-32.

19. Body JJ, Casimiro S, Costa L. Targeting bone metastases in prostate cancer: improving clinical outcome. Nat Rev Urol 2015;12:340-56.

20. Xiang L, Gilkes DM. The contribution of the immune system in bone metastasis pathogenesis. Int J Mol Sci 2019;20:999.

21. Batlle E, Massagué J. Transforming growth factor- $\beta$ signaling in immunity and cancer. Immunity 2019;50:924-40.

22. Cook LM, Shay G, Araujo A, et al. Integrating new discoveries into the "vicious cycle" paradigm of prostate to bone metastases. Cancer Metastasis Rev 2014;33:511-25.

23. Jiao S, Subudhi SK, Aparicio A, et al. Differences in tumor microenvironment dictate $\mathrm{T}$ helper lineage polarization 
and response to immune checkpoint therapy. Cell 2019;179:1177-90.e13.

24. Beer TM, Kwon ED, Drake CG, et al. Randomized, double-blind, phase III trial of ipilimumab versus placebo in asymptomatic or minimally symptomatic patients with metastatic chemotherapy-naive castration-resistant prostate cancer. J Clin Oncol 2017;35:40-7.

25. Korn T, Bettelli E, Oukka M, et al. IL-17 and Th17 cells. Annu Rev Immunol 2009;27:485-517.

26. Gaffen SL, Jain R, Garg AV, et al. The IL-23-IL-17 immune axis: from mechanisms to therapeutic testing. Nat Rev Immunol 2014;14:585-600.

27. Mariathasan S, Turley SJ, Nickles D, et al. TGF $\beta$ attenuates tumour response to $\mathrm{PD}-\mathrm{L} 1$ blockade by contributing to exclusion of T cells. Nature 2018;554:544-8.

28. Tauriello DVF, Palomo-Ponce S, Stork D, et al. TGF $\beta$ drives immune evasion in genetically reconstituted colon cancer metastasis. Nature 2018;554:538-43.

29. Robinson D, Van Allen EM, Wu YM, et al. Integrative clinical genomics of advanced prostate cancer. Cell 2015;162:454.

30. Ylitalo EB, Thysell E, Jernberg E, et al. Subgroups of castration-resistant prostate cancer bone metastases defined through an inverse relationship between androgen receptor activity and immune response. Eur Urol 2017;71:776-87.
Cite this article as: Fonseca NM, Roberts ME, Wyatt AW. A marrow-minded look at immune checkpoint blockade resistance in metastatic castration resistant prostate cancer. Transl Androl Urol 2021;10(10):4009-4013. doi: 10.21037/tau-20-1205 